\title{
Induced Chern-Simons action on noncommutative torus
}

\author{
D. V. Vassilevich* \\ Instituto de Física, Universidade de São Paulo, \\ Caixa Postal 66318 CEP 05315-970, São Paulo, S.P., Brazil
}

August 28, 2018

\begin{abstract}
We compute a Chern-Simons term induced by the fermions on noncommutative torus interacting with two $U(1)$ gauge fields. For rational noncommutativity $\theta \propto P / Q$ we find a new mixed term in the action which involves only those fields which are $(2 \pi) / Q$ periodic, like the fields in a crystal with $Q^{2}$ nodes.
\end{abstract}

It is known since a long time that quantum one-loop corrections due to 3dimensional fermions generate the Chern-Simons action [1-3]. This fact has far reaching physical consequences, see [4] for a review. It is natural to consider this mechanism in the framework of noncommutative (NC) field theorie. This was done in $[5,6]$ on the 3-dimensional Moyal plane. Different properties of NC Chern-Simons theories were studied in a number of publications, see, e.g., [7] and references therein.

The main novelty of the present work is that we consider the Dirac fermions on a compact $\mathrm{NC}$ manifold (an NC 3-torus) which are coupled to two independent $U(1)$ fields which act by left and right Moyal multiplications. We calculate the parity-violating part of the effective action (which generalizes the Chern-Simons action for this case). We find a mixed contribution to this action. For a rational NC parameter, $\theta /(2 \pi)=P / Q$ this mixed term exhibits a very interesting property: it involves interactions only between the fields which are $(2 \pi) / Q$-periodic in the $\mathrm{NC}$ directions. We see a kind of dynamical crystallization of the torus due to the effects of $\mathrm{NC}$ quantum field theory.

In this work we use the zeta-function regularization and the heat-kernel methods (applied earlier to commutative low-dimensional fermionic systems in e.g.

*On leave from V. A. Fock Institute of Physics, St. Petersburg University, Russia. E.mail: dmitry@dfn.if.usp.br 
$[8,9])$. Physical and mathematical aspects of this very powerful machinery are reviewed in $[10,11]$. For the Moyal-type noncommutativity the heat kernel expansion was constructed in [12-15].

The Moyal star-product on $\mathbb{T}^{3}$ is defined as usual by

$$
f_{1} \star f_{2}(x)=\left.\exp \left(\frac{i}{2} \Theta^{\mu \nu} \partial_{\mu}^{x} \partial_{\nu}^{y}\right) f_{1}(x) f_{2}(y)\right|_{y=x} .
$$

The constant noncommutativity parameter $\Theta$ is an antisymmetric $3 \times 3$ matrix which is inevitably degenerate 1 . Our principle example is

$$
\Theta^{12}=-\Theta^{21} \equiv \theta, \quad \Theta^{13}=\Theta^{23}=0 .
$$

Main formulae will be valid for generic $\Theta^{\mu \nu}$, but the choice (2) makes the results most transparent.

We take classical action for the Dirac fermions in the form

$$
S=\int d^{3} x \sqrt{g} \bar{\psi} \not D \psi
$$

where

$$
\not D=i \gamma^{\mu}\left(\partial_{\mu}+i L\left(A_{\mu}^{L}\right)+i R\left(A_{\mu}^{R}\right)\right)
$$

Here $L$ and $R$ are left and right Moyal multiplications, $L(f) \phi=f \star \phi, R(f) \phi=$ $\phi \star f$. Formal adjoints of these operators coincide with multiplications by complex conjugate functions, e.g. $L(f)^{\dagger}=L\left(f^{*}\right)$. It is convenient to keep the ranges for all coordinates $x^{\mu}$ on the torus $\mathbb{T}^{3}$ from 0 to $2 \pi$, but to allow for a constant Euclidean metric $g_{\mu \nu}$. The $\gamma$-matrices are defined by the condition $\gamma^{\mu} \gamma^{\nu}+\gamma^{\nu} \gamma^{\mu}=2 g^{\mu \nu}$, and $\operatorname{tr} \gamma^{\mu} \gamma^{\nu} \gamma^{\rho}=2 i \epsilon^{\mu \nu \rho}$ with $\epsilon^{123}=g^{-1 / 2}$. To simplify our discussion we impose periodic boundary conditions on the fermions on $\mathbb{T}^{3}$. A short discussion of antiperiodic boundary conditions is postponed until the end of this Letter.

As compared to previous works on induced NC Chern-Simons theory $[5,6]$ we have two independent vector fields instead of one. The case $A_{\mu}^{R}=0$ corresponds to gauge fields in the fundamental representation in the terminology of [6] or to the Dirac fermions in the terminology of [5]. $A_{\mu}^{L}=0$ corresponds to antifundamental gauge fields [6], and $A_{\mu}^{R}=-A_{\mu}^{L}$ is the adjoint representation [6] or Majorana fermions [5]. There are two gauge symmetries 2 corresponding to two $U(1)$ gauge fields (so that we have a double gauging of $U(1)[18]$ ):

$$
\begin{aligned}
& \psi \rightarrow U_{L} \star \psi \star U_{R}, \quad \bar{\psi} \rightarrow U_{R}^{\dagger} \star \bar{\psi} \star U_{L}^{\dagger}, \\
& i A_{\mu}^{L} \rightarrow U_{L} \star \partial_{\mu} U_{L}^{-1}+i U_{L} \star A_{\mu}^{L} \star U_{L}^{-1}, \\
& i A_{\mu}^{R} \rightarrow \partial_{\mu} U_{R}^{-1} \star U_{R}+i U_{R}^{-1} \star A_{\mu}^{R} \star U_{R},
\end{aligned}
$$

\footnotetext{
${ }^{1}$ On noncompact NC manifolds degenerate $\Theta$ may cause problems in quantum theory [16]. This is one of the reasons to prefer the NC torus over the NC plane in this work.

${ }^{2}$ General discussion of gauge symmetris compartible with noncommutativity can be found in $[17]$.
} 
where $U_{L, R}$ are star-unitary, $U_{L, R} \star U_{L, R}^{\dagger}=1$. The Dirac operator is transformed as $\not D \rightarrow R\left(U_{R}\right) L\left(U_{L}\right) \not D L\left(U_{L}^{-1}\right) R\left(U_{R}^{-1}\right)$. Because of these two symmetries there are two independent currents, $j_{L}^{\mu}=\psi^{b} \star \bar{\psi}^{a} \gamma_{a b}^{\mu}$ and $j_{R}^{\mu}=\bar{\psi}^{a} \star \psi^{b} \gamma_{a b}^{\mu}$ (with $a, b$ being spinor indices) which are separately covariantly conserved

$$
\partial_{\mu} j_{L}^{\mu}+i A_{\mu}^{L} \star j_{L}^{\mu}-i j_{L}^{\mu} \star A_{\mu}^{L}=0, \quad \partial_{\mu} j_{R}^{\mu}-i A_{\mu}^{R} \star j_{R}^{\mu}+i j_{R}^{\mu} \star A_{\mu}^{R}=0 .
$$

The existence of two independent vector currents is an additional motivation to introduce two vector field coupled to them, which is even necessary if one uses such vectors to study the dynamics of collective excitations of the fermions.

The Dirac operator squared is an operator of Laplace type, i.e.

$$
\begin{aligned}
& \not D^{2}=-\left(\nabla^{2}+E\right), \\
& \nabla_{\mu}=\partial_{\mu}+i L\left(A_{\mu}^{L}\right)+i R\left(A_{\mu}^{R}\right), \\
& E=\frac{i}{2}\left[\gamma^{\mu}, \gamma^{\nu}\right]\left(L\left(\partial_{\mu} A_{\nu}^{L}+i A_{\mu}^{L} \star A_{\nu}^{L}\right)+R\left(\partial_{\mu} A_{\nu}^{R}+i A_{\nu}^{R} \star A_{\mu}^{R}\right)\right) .
\end{aligned}
$$

We are going to use the heat-kernel methods, so let us remind some basic facts [15] regarding the heat kernel expansion on $\mathrm{NC}$ torus for an operator of Laplace type containing both left and right Moyal multiplications. The net result of [15] is that the heat kernel expansion looks precisely as in the commutative case if one uses a modified trace operation. To define this trace we need a little bit of number theory. The construction will be given in all detail for the special case of (2). For generic $\Theta^{\mu \nu}$ the reader can consult [15]. A real number $\alpha$ is called Diophantine if there are two positive constants $C$ and $\beta$ such that

$$
\inf _{P \in \mathbb{Z}}|\alpha Q-P| \geq \frac{C}{|Q|^{1+\beta}} \quad \text { for all } \quad Q \in \mathbb{Z} .
$$

In other words, the Diophantine numbers are the numbers which cannot be too well approximated by rational numbers. We suppose that $(\theta / 2 \pi)$ is either rational, or Diophantine 3 .

Next we define a special subset $\mathcal{Z}$ of the Fourier momenta. A momentum $q \in \mathbb{Z}^{3}$ belongs to $\mathcal{Z}$ iff

$$
(2 \pi)^{-1} \Theta q \in \mathbb{Z}^{3} .
$$

(This formula is also valid for generic $\Theta^{\mu \nu}$ ). Let us give some examples. In the commutative case, $\theta=0$, the condition (9) is satisfied by all momenta, and $\mathcal{Z}=\mathbb{Z}^{3}$. If $\theta /(2 \pi)$ is irrational (then under our assumptions it is also Diophantine), only the $q_{3}$ can be non-zero, and $\mathcal{Z}=\{0\} \otimes\{0\} \otimes \mathbb{Z}$. The most interesting case is rational noncommutativity. Then

$$
\mathcal{Z}=Q \cdot \mathbb{Z} \otimes Q \cdot \mathbb{Z} \otimes \mathbb{Z} \quad \text { for } \quad \theta /(2 \pi)=P / Q
$$

\footnotetext{
${ }^{3}$ If $(\theta / 2 \pi)$ is neither this nor that, the heat kernel asymptotics are unstable, i.e. the powers of the proper time appearing in the asymptotic expansion depend on the ultra-violet behavior of the background fields, see App. B of [15]. The fact that quantum field theory on NC torus is very sensitive to the number theory nature of $\theta$ was noted already in [19].
} 
with $P \in \mathbb{Z}$ and $Q \in \mathbb{N}$. (Of course, $P / Q$ must be irreducible). The set $\mathcal{Z}$ depends on $Q$ but not on $P$. Note that two previous cases may be obtained by taking formal limits $Q \rightarrow 1$ and $Q \rightarrow \infty$ in (10), respectively. In the sense of this remark $\mathcal{Z}$ is uniquely defined by a number $Q \in \mathbb{N} \cup\{\infty\}$.

Let us now define the trace. Consider an operator which can be represented as a product of left and right Moyal multiplications $L(l) R(r)$, possibly with some matrix structures. Then

$$
\operatorname{Sp}(L(l) R(r))=\sqrt{g} \sum_{q \in \mathcal{Z}} \tilde{l}(-q) \tilde{r}(q)
$$

where $\tilde{l}$ and $\tilde{r}$ are the Fourier modes:

$$
\tilde{l}(k)=(2 \pi)^{-3 / 2} \int d^{3} x l(x) e^{-i k x} .
$$

The definition (11) includes also trace over all matrix indices, which we do not write explicitly here.

Now consider an operator $P$ on $\mathrm{NC} \mathbb{T}^{n}$ which can be represented as $P=$ $-\left(\nabla^{2}+E\right)$ where $E$ and $\omega_{\mu}$ in $\nabla_{\mu}=\partial_{\mu}+\omega_{\mu}$ are zeroth order operators, i.e., $E$ and $\omega_{\mu}$ are combinations of left and right Moyal multiplications. Such operators are called generalized Laplacians $\left(\not D^{2}\right.$ is an example, see eq. (17)). It was demonstrated in [15] that for such operators the heat operator $e^{-t P}$ exists for positive $t$ and is trace class, and there is a full asymptotic series as $t \rightarrow+0$

$$
\operatorname{Tr}\left(L(l) R(r) e^{-t P}\right) \simeq \sum_{m=0}^{\infty} t^{(n-m) / 2} a_{2 m}(L(l) R(r), P)
$$

Tr is the $L_{2}$ trace. In particular, first couple of the heat kernel coefficient: 4 read

$$
\begin{aligned}
& a_{0}=(4 \pi)^{-n / 2} \operatorname{Sp}(L(l) R(r)), \\
& a_{2}=(4 \pi)^{-n / 2} \operatorname{Sp}(L(l) R(r) E) .
\end{aligned}
$$

It is easy to figure out how the eqs. (9) and (12) must be generalized to arbitrary $n$.

In this Letter we employ the zeta-function regularization which a is a proper instrument to keep gauge invariance throughout the calculations [20]. We first use the relation $[2,3,20]$ between the parity-violating part of the effective action and the eta invariant

$$
\Gamma^{\mathrm{pv}}=i \frac{\pi}{2} \eta(0)
$$

\footnotetext{
${ }^{4}$ We use "inflated notations" for the heat kernel coefficients, so that only even-numbered coefficients appear usually on manifolds without a boundary. In this nomenclature no halfinteger indices appear also in the presence of boundaries, see [10,11].
} 
which is defned through a sum over the eigenvalues of $\not D$,

$$
\eta(s)=\sum_{\lambda_{n}>0}\left(\lambda_{n}\right)^{-s}-\sum_{\lambda_{n}<0}\left(-\lambda_{n}\right)^{-s}
$$

This spectral function measures the spectral asymmetry of the Dirac operator. Next couple of steps repeat quite literally those of [2]. We make use of an integral representation of the eta function and replace the sum over the spectrum by Tr.

$$
\eta(s)=\frac{2}{\Gamma((s+1) / 2)} \int_{0}^{\infty} d \tau \tau^{s} \operatorname{Tr}\left(\not D e^{-\tau^{2} \not D^{2}}\right) .
$$

Let us vary $A_{\mu}^{L, R}$ in $\not D$. The variation of $\eta(s)$ reads

$$
\delta \eta(s)=\frac{2}{\Gamma((s+1) / 2)} \int_{0}^{\infty} d \tau \tau^{s} \frac{d}{d \tau} \operatorname{Tr}\left((\delta \not D) \tau e^{-\tau^{2} \not D^{2}}\right) .
$$

Now, by taking $s \rightarrow 0$ (and assuming that the heat kernel decays fast enough at $\tau^{2} \rightarrow \infty$, which is usually true) one arrives at

$$
\begin{aligned}
\delta \eta(0) & =-\frac{2}{\sqrt{\pi}} \lim _{\tau \rightarrow 0} \operatorname{Tr}\left((\delta \not D) \tau e^{-\tau^{2} \not D^{2}}\right) \\
& =-\frac{2}{\sqrt{\pi}} \lim _{t \rightarrow 0} \operatorname{Tr}\left((\delta \not D) t^{1 / 2} e^{-t \not D^{2}}\right) .
\end{aligned}
$$

To evaluate this limit we use the heat kernel expansion (13). The coefficient $a_{0}$ does not contribute because of the $\gamma$-trace. We are left with

$$
\delta \eta(0)=-\frac{2}{\sqrt{\pi}} a_{2}\left(\delta \not D, \not D D^{2}\right)
$$

This heat kernel coefficient reads

$$
a_{2}\left(\delta \not D, \not D^{2}\right)=\frac{1}{8 \pi^{3 / 2}} \mathrm{Sp}_{2}\left(\gamma^{\mu}\left(L\left(-\delta A_{\mu}^{L}\right)+R\left(-\delta A_{\mu}^{R}\right)\right) \cdot E\right)
$$

where $E$ is given by (17) and the subscript "2" in $\mathrm{Sp}_{2}$ reminds to calculate the trace over the spinor indices which yields

$$
\begin{aligned}
& a_{2}\left(\delta \not D, \not D^{2}\right)=\frac{1}{4 \pi^{3 / 2}} \epsilon^{\mu \nu \rho} \operatorname{Sp}\left(\left(L\left(\delta A_{\mu}^{L}\right)+R\left(\delta A_{\mu}^{R}\right)\right)\right. \\
& \cdot\left(L\left(\partial_{\nu} A_{\rho}^{L}+i A_{\nu}^{L} \star A_{\rho}^{L}\right)+R\left(\partial_{\nu} A_{\rho}^{R}+i A_{\rho}^{R} \star A_{\nu}^{R}\right)\right)
\end{aligned}
$$

To calculate the remaining trace we need a couple of relations. First,

$$
\operatorname{Sp}(L(f))=\operatorname{Sp}(R(f))=\int d^{3} x \sqrt{g} f(x)
$$


where to apply the definition (11) one has to write $L(f)=L(f) R(1)$. Next, we have the symmetry property

$$
\begin{aligned}
& \operatorname{Sp}\left(L(f) R\left(f_{1} \star f_{2}\right)\right)=\operatorname{Sp}\left(L(f) R\left(f_{2} \star f_{1}\right)\right) \\
& \operatorname{Sp}\left(L\left(f_{1} \star f_{2}\right) R(f)\right)=\operatorname{Sp}\left(L\left(f_{2} \star f_{1}\right) R(f)\right)
\end{aligned}
$$

for any functions $f, f_{1}, f_{2}$. The relations (24) and (25) were derived in [15].

To derive another useful property we should first define a $Q$-periodic projection of functions on $\mathbb{T}^{3}$

$$
[f]_{Q}:=(2 \pi)^{-3 / 2} \sum_{k \in \mathcal{Z}} \tilde{f}(k) e^{i k x}
$$

(For generic $\Theta^{\mu \nu}$ one can define a projection $[f]_{\mathcal{Z}}$; the right hand side of (26) remains unchanged). This is indeed a projection, $\left[[f]_{Q}\right]_{Q}=[f]_{Q}$. For a rational $\theta /(2 \pi)=P / Q$ this operation selects a part of $f$ which is periodic in $x^{1}$ and $x^{2}$ coordinates with the period $2 \pi / Q$. In the irrational (Diophantine) case this operation (which may be denoted as $[f]_{\infty}$ according to the remark below eq. (10)) selects just the average value of $f$ on the $\mathbb{T}^{2}$ spanned by $x^{1}$ and $x^{2}$. In the commutative case, $\mathcal{Z}=\mathbb{Z}^{3}$, this is the identity map $[f]_{1}=f$. By making the Fourier transform back and forth one can prove that

$$
\operatorname{Sp}(L(l) R(r))=\int_{\mathbb{T}^{3}} d^{3} x \sqrt{g}[l]_{Q} \cdot[r]_{Q}=\int_{\mathbb{T}^{3}} d^{3} x \sqrt{g}[l]_{Q} \star[r]_{Q}
$$

By using (24), (25) and (27) we rewrite (23) as

$$
\begin{aligned}
& a_{2}\left(\delta \not D, \not D^{2}\right)=a_{2}^{L}+a_{2}^{R}+a_{2}^{\text {mixed }} \\
& a_{2}^{L}=4 \pi^{-3 / 2} \int d^{3} x \sqrt{g} \epsilon^{\mu \nu \rho}\left(\delta A_{\mu}^{L}\right)\left(\partial_{\nu} A_{\rho}^{L}+i A_{\nu}^{L} \star A_{\rho}^{L}\right) \\
& a_{2}^{R}=4 \pi^{-3 / 2} \int d^{3} x \sqrt{g} \epsilon^{\mu \nu \rho}\left(\delta A_{\mu}^{R}\right)\left(\partial_{\nu} A_{\rho}^{R}-i A_{\nu}^{R} \star A_{\rho}^{R}\right) \\
& a_{2}^{\text {mixed }}=4 \pi^{-3 / 2} \int d^{3} x \sqrt{g} \epsilon^{\mu \nu \rho}\left(\left[\left(\delta A_{\mu}^{L}\right)\right]_{Q} \partial_{\nu}\left[A_{\rho}^{R}\right]_{Q}+\left[\left(\delta A_{\mu}^{R}\right)\right]_{Q} \partial_{\nu}\left[A_{\rho}^{L}\right]_{Q}\right) .
\end{aligned}
$$

Cubic terms in $a_{2}^{\text {mixed }}$ vanish due to (25).

In the zeta function regularization (see, e.g. [8]) the parity-violating part of the effective action $\Gamma^{\mathrm{pv}}$ is expressed through the Chern-Simons action $S_{\mathrm{CS}}$ by means of the relation $\Gamma^{\mathrm{pv}}=\frac{1}{2} S_{\mathrm{CS}}$. (In the Pauli-Villars regularization, for examples, the PV masses also enter this relation). We combine (16) and (21) with (28) to obtain a generalized Chern-Simons action induced by fermionic fluctuations on 
the NC torus

$$
\begin{aligned}
& S_{\mathrm{CS}}=S_{\mathrm{CS}}^{L}+S_{\mathrm{CS}}^{R}+S_{\mathrm{CS}}^{\text {mixed }} \\
& S_{\mathrm{CS}}^{L}=-\frac{i}{4 \pi} \int d^{3} x \sqrt{g} \epsilon^{\mu \nu \rho}\left(A_{\mu}^{L} \partial_{\nu} A_{\rho}^{L}+\frac{2 i}{3} A_{\mu}^{L} \star A_{\nu}^{L} \star A_{\rho}^{L}\right), \\
& S_{\mathrm{CS}}^{R}=-\frac{i}{4 \pi} \int d^{3} x \sqrt{g} \epsilon^{\mu \nu \rho}\left(A_{\mu}^{R} \partial_{\nu} A_{\rho}^{R}-\frac{2 i}{3} A_{\mu}^{R} \star A_{\nu}^{R} \star A_{\rho}^{R}\right), \\
& S_{\mathrm{CS}}^{\text {mixed }}=-\frac{i}{2 \pi} \int d^{3} x \sqrt{g} \epsilon^{\mu \nu \rho}\left[A_{\mu}^{L}\right]_{Q} \partial_{\nu}\left[A_{\rho}^{R}\right]_{Q} .
\end{aligned}
$$

Note, that the combination $\sqrt{g} \epsilon^{\mu \nu \rho}$ does not depend on the metric, so that $S_{\mathrm{SC}}$ is also metric-independent (topological) as in the commutative case.

The "planar" terms (30) and (31) are pretty much standard NC generalizations of the Chern-Simons action in all particular cases considered in [5,6] (cf. also the discussion above eq. (50)). The reason is that the analytic expressions for planar heat kernel coefficients are not sensitive to the space-time topology. There is one particular case, $A^{L}=A^{R}$, when all noncommuativity effects disappear from the planar contributions. However, there is no $U(1)$ subgroup of the gauge transformations (5) which respects the choice $A^{L}=A^{R}$, so this case is not interesting.

The mixed term (32) exhibits a rather interesting physical property. It involves interactions only between those background fields $A_{\mu}^{L, R}$ which are $(2 \pi) / Q$ periodic in the $x^{1}$ and $x^{2}$ directions. Such fields remind us of solid state physics and correspond to a crystal consisting of $Q \times Q$ fundamental domains. By stretching a bit the terminology we can say that the two-torus is dynamically crystallized due to NC quantum effects. It would be very interesting to establish precise relations between this effect and the works on the equivalence of field theories on NC torus with ratioanal noncommutativity to matrix models (see, e.g., [21]).

When using the spectral geometry methods one should not worry too much about the gauge invariance since the eta function (17) is manifestly gauge invariant. One can also check the gauge invariance of (30) - (32) by direct calculations. To this end the relation (25) is very useful.

The Chern-Simons action (29) does not depend smoothly on $\theta$. Moreover, we have calculated this action for a rational or Diophantine noncommutativity only. Nevertheless, there is a well defined commutative limit, though not an obvious one. Instead of taking $\theta \rightarrow 0$, we take a rational $\mathrm{NC}$ parameter with $Q=1$. Then the star-product becomes commutative (one easily gets: $e^{i k x} \star e^{i q x}=e^{i q x} \star e^{i k x}=$ $\pm e^{i(k+q) x}$ ), though still not the ordinary one (unless $P$ is even). Let us introduce two new vector fields by $A^{L}=\frac{1}{2}(B+C), A^{R}=\frac{1}{2}(B-C)$. We have

$$
\left.S_{\mathrm{CS}}\right|_{Q=1}=-\frac{i}{4 \pi} \int d^{3} x \sqrt{g} \epsilon^{\mu \nu \rho} B_{\mu} \partial_{\nu} B_{\rho} .
$$

We see, that, precisely as one would expect in the commutative limit, the field $C_{\mu}$ disappears, and the action for $B_{\mu}$ is the standard abelian Chern-Simons action. 
To be able to use the heat kernel asymptotics from [15] we assumed that $\psi$ is periodic on the torus. If instead one chooses (perhaps physically more preferable) anti-periodic boundary conditions in some of the coordinates, then allowed fermionic momenta in corresponding directions are shifted by $1 / 2$ to form a set which we call $\widetilde{\mathbb{Z}}^{3}$ instead of $\mathbb{Z}^{3}$ considered above. The definition

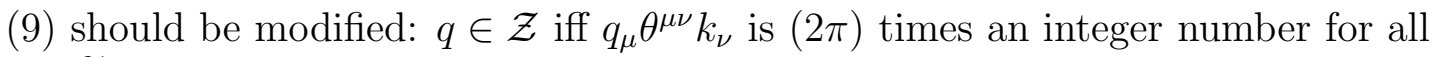
$k \in \widetilde{\mathbb{Z}}^{3}$. (Note that $\mathcal{Z} \subset \mathbb{Z}^{3}$ as before since $q$ 's are the Fourier momenta of bosonic background fields). This is the most important modification, which, of course, also leads to some changes in the periodic projections. Qualitatively our results remain unchanged. We hope to consider anti-periodic boundary conditions in more detail in a future publication.

To summarize, in this Letter we calculated the Chern-Simons action induced by the parity anomaly of fermions on $\mathrm{NC} 3$-torus. Due to the compactnes: 5 of the manifold and due to the presence of two independent gauge fields we found that there is a mixed (non-planar) contribution to the action of a rather particular form (32). This mixed term depends only on the fields which are $(2 \pi) / Q$-periodic and thus remind us of the fields in a crystal. It would be interesting to test this result at physical applications of the NC Chern-Simons, e.g. at the model [22] of Quantum Hall Fluids.

\section{References}

[1] A. N. Redlich, Phys. Rev. Lett. 52 (1984) 18; Phys. Rev. D 29 (1984) 2366. A. N. Redlich and L. C. R. Wijewardhana, Phys. Rev. Lett. 54 (1985) 970. A. J. Niemi and G. W. Semenoff, Phys. Rev. Lett. 54 (1985) 2166.

[2] L. Alvarez-Gaume, S. Della Pietra and G. W. Moore, Annals Phys. 163 (1985) 288.

[3] A. J. Niemi, Phys. Rev. Lett. 57 (1986) 1102.

[4] G. V. Dunne, "Aspects of Chern-Simons theory," arXiv:hep-th/9902115.

[5] C. S. Chu, Nucl. Phys. B 580 (2000) 352 arXiv:hep-th/0003007].

[6] N. E. Grandi and G. A. Silva, Phys. Lett. B 507 (2001) 345 arXiv:hep-th/0010113.

[7] E. A. Asano, L. C. T. Brito, M. Gomes, A. Y. Petrov and A. J. da Silva, Phys. Rev. D 71 (2005) 105005 [arXiv:hep-th/0410257].

\footnotetext{
${ }^{5}$ To see that the compactness is essential it is enough to compare the heat kernel expansions on the Moyal plane [14] and on the Moyal torus [15].
} 
[8] R. E. Gamboa Saravi, M. A. Muschietti, F. A. Schaposnik and J. E. Solomin, J. Math. Phys. 26 (1985) 2045.

[9] R. E. Gamboa Saravi, G. L. Rossini and F. A. Schaposnik, Int. J. Mod. Phys. A 11 (1996) 2643 arXiv:hep-th/9411238.

[10] P. B. Gilkey, Asymptotic formulae in spectral geometry, Chapman \& Hall/CRC, Boca Raton, FL, 2004.

K. Kirsten, Spectral functions in mathematics and physics, Chapman \& Hall/CRC, Boca Raton, FL, 2001.

[11] D. V. Vassilevich, Phys. Rept. 388 (2003) 279 arXiv:hep-th/0306138.

[12] D. V. Vassilevich, Lett. Math. Phys. 67 (2004) 185 arXiv:hep-th/0310144.

[13] V. Gayral and B. Iochum, J. Math. Phys. $46 \quad$ (2005) 043503 arXiv:hep-th/0402147.

[14] D. V. Vassilevich, JHEP 0508 (2005) 085 arXiv:hep-th/0507123.

[15] V. Gayral, B. Iochum and D. V. Vassilevich, "Heat kernel and number theory on NC-torus," arXiv:hep-th/0607078, to appear in Commun. Math. Phys.

[16] V. Gayral, J. M. Gracia-Bondia and F. R. Ruiz, Phys. Lett. B 610 (2005) 141 arXiv:hep-th/0412235.

[17] M. Chaichian, P. Presnajder, M. M. Sheikh-Jabbari and A. Tureanu, Phys. Lett. B 526 (2002) 132 arXiv:hep-th/0107037.

[18] Y. Liao and K. Sibold, Phys. Lett. B 586 (2004) 420 [arXiv:hep-th/0401105].

[19] T. Krajewski and R. Wulkenhaar, Int. J. Mod. Phys. A 15 (2000) 1011 arXiv:hep-th/9903187.

[20] S. Deser, L. Griguolo and D. Seminara, Phys. Rev. Lett. 79 (1997) 1976 arXiv:hep-th/9705052; Phys. Rev. D 57 (1998) 7444 arXiv:hep-th/9712066.

[21] I. Bars and D. Minic, Phys. Rev. D 62 (2000) 105018 arXiv:hep-th/9910091]. J. Ambjorn, Y. M. Makeenko, J. Nishimura and R. J. Szabo, JHEP 0005 (2000) 023 arXiv:hep-th/0004147.

M. Chaichian, A. Demichev, P. Presnajder, M. M. Sheikh-Jabbari and A. Tureanu, Nucl. Phys. B 611 (2001) 383 arXiv:hep-th/0101209.

[22] L. Susskind, "The quantum Hall fluid and non-commutative Chern Simons theory," arXiv:hep-th/0101029.

J. L. F. Barbon and A. Paredes, Int. J. Mod. Phys. A 17 (2002) 3589 arXiv:hep-th/0112185]. 